\title{
Improved microscopy with ultraviolet surface excitation (MUSE) using high-index immersion illumination
}

\author{
Vincent D. Ching-RoA, (1) Chi Z. Huang, And Michael G. \\ GIACOMELLI* \\ Department of Biomedical Engineering, University of Rochester, 207 Goergen Hall, Box 270168, Rochester, \\ NY 14627, USA \\ *mgiacome@ur.rochester.edu
}

\begin{abstract}
Microscopy with ultraviolet surface excitation (MUSE) typically has an optical sectioning thickness significantly larger than standard physical sectioning thickness, resulting in increased background fluorescence and higher feature density compared to formalin-fixed, paraffin-embedded physical sections. We demonstrate that high-index immersion with angled illumination significantly reduces optical sectioning thickness through increased angle of refraction of excitation light at the tissue interface. We present a novel objective dipping cap and waveguide-based MUSE illuminator design with high-index immersion and quantify the improvement in optical sectioning thickness, demonstrating an $\mathrm{e}^{-1}$ section thickness reduction to $6.67 \mu \mathrm{m}$ in tissue. Simultaneously, the waveguide illuminator can be combined with high or low magnification objectives, and we demonstrate a $6 \mathrm{~mm}^{2}$ field of view, wider than a conventional 10x pathology objective. Finally, we show that resolution and contrast can be further improved using deconvolution and focal stacking, enabling imaging that is robust to irregular surface profiles on surgical specimens.
\end{abstract}

(C) 2021 Optical Society of America under the terms of the OSA Open Access Publishing Agreement

\section{Introduction}

Conventional formalin-fixed, paraffin-embedded (FFPE) histology, based on physical sectioning of tissue specimens into optically thin slices, remains the current standard of care for assessment of surgical specimens, biopsies, and most excised tissue specimens. With physical sectioning, thin sections ( $\sim 5 \mu \mathrm{m}$ thick) of tissue are cut using a microtome and mounted on glass slides in order to be viewed with a bright-field transmission microscope. However, while FFPE produces high-quality sections, processing is time and labor-intensive, with slides typically available after at least a day's delay. For more time-critical operations such as with intraoperative margin evaluations, day-long delays are not acceptable and frozen section (FS) histology may be utilized. FS processing takes a shorter time (20-30 minutes) by replacing paraffin embedding with rapid freezing, but is prone to freezing artifacts and difficult to perform on many tissue types [1,2], while requiring expensive equipment with highly skilled technicians to perform delicate sectioning. Recent developments in microscopy have given rise to techniques that replace physical sectioning with optical sectioning, extracting virtual histology sections from a larger 3D tissue specimen with little to no processing. These new techniques dramatically reduce time and skill requirements, and therefore are attractive alternatives to FS.

Techniques such as confocal fluorescence microscopy [3-5], two-photon excitation fluorescence microscopy [6-9], stimulated Raman scattering microscopy [10], structured illumination microscopy [11], and light sheet microscopy [12,13] take advantage of non-destructive optical sectioning for virtual histology. While these approaches often offer high resolution and good penetration depth into intact tissues, they require relatively complex and costly optical setups with either femtosecond lasers, scanning optics, optical modulators, and/or multiple objectives. 
Microscopy with ultraviolet surface excitation (MUSE) is a recently developed technique by Levenson and Demos that allows for optical sectioning through the use of deep ultraviolet (DUV) light with wavelength shorter than $300 \mathrm{~nm}$ [14-16]. DUV is known to be strongly absorbed by DNA, carbohydrates, and structural proteins which constitute most non-water components of soft tissue $[17,18]$. The strong optical absorption results in the excitation light being confined to the tissue surface, localizing fluorescence excitation to a thin layer which is analogous to section thickness in conventional paraffin or frozen section histology. Compared to other techniques, optical sectioning with DUV illumination is extremely inexpensive and simple. Furthermore, as no scanning is required, imaging throughput can potentially extend beyond what is possible with sequential point scanning techniques. As a result, MUSE has the potential to be an accessible, high-throughput tool for histological applications such as with cancer biopsy [19,20], or surgical margin examination [21,22] wherein only the surface of the excision is of the main interest.

Previous work by Yoshitake et al. have shown that effective image resolution and interpretability are highly dependent on thickness of the optical section provided by the system illumination [19]. Furthermore, optical sectioning thickness imposes a minimum limit on the system's depth of field which should be greater than or equal to the optical sectioning thickness to avoid blurring from out-of-focus depths. Because an increase in numerical aperture leads to a decrease in depth of field, there is a maximum numerical aperture that matches to the minimum depth of field. This limit in numerical aperture in turn limits image lateral resolution. Computational methods have been used to extend depth-of-field, such as with wavefront coding [23], focal stacking $[15,21]$ or deep-learning [24]. More specifically, work by Xie et al. have shown the use of both deconvolution and focus stacking to reconstruct a full image mosaic with improved contrast and resolution while resolving irregular surface profiles with extended depth of field [21]. While computational solutions can extend depth of field, a more fundamental issue faced by a thick section is that despite being completely resolved, the image will have volumetric data axially integrated onto a two-dimensional sensor. This results in denser, more homogeneous histological features and increased cellularity compared to that of FFPE or frozen sections, making it harder to interpret key features in histology $[15,19,21]$. In contrast to computationally extending depth of field, decreasing optical sectioning thickness improves feature density, increases contrast and reduces background fluorescence.

In most MUSE systems, angled illumination is implemented both to decouple the DUV light optical path from the detection optics and to reduce optical sectioning thickness [15,19,21]. However, the minimum optical sectioning thickness achieved through just angled illumination is limited by refraction at the tissue surface, which has been evaluated previously by Yoshitake et al. to be approximately $20 \mu \mathrm{m}$ in breast tissue [19]. This minimum limit can be further decreased by adding water immersion with angled illumination, resulting in a reduction of $\sim 10 \mu \mathrm{m}$ in breast tissue from the same study. Similar minimum optical sectioning thickness has been reached with frustrated total internal reflection (FTIR) systems where illumination is directly coupled into a thick optical window [25]. Compared to physical sectioning thicknesses in conventional histology, $10 \mu \mathrm{m}$ sectioning thickness is still relatively thick, and such will result in image interpretability issues for some tissue types such as with breast or skin [19].

Our improved MUSE system builds upon these previous works by both decreasing the optical sectioning thickness close to the standard $5 \mu \mathrm{m}$ using high-index immersion angled illumination and combining that with deconvolution and focus stacking to yield H\&E slide-like images with high resolution and contrast while possessing capability for high field-of-view operation. We demonstrate the use of a high-index immersion objective dipping cap and waveguide-based illuminator to decrease the optical sectioning thickness and then characterize the improvement in resolution. Compared to previous work with water immersion MUSE where the illumination and objective immersion media are the same [19], this approach allows for independent control of illumination and objective media. This enables us to have a large field of view, low optical 
sectioning thickness MUSE system using a low magnification air objective and glycerol as our illumination immersion media. We also show that our MUSE system also gains improved image resolution and robustness from surface irregularities with deconvolution and focal stacking.

\section{Methods}

\subsection{Surgical specimens and fluorescence staining}

Discarded frozen skin tissue were acquired under a protocol approved by the Research Subjects Review Board at the University of Rochester Medical Center. Thawed specimens were stained for 5 minutes with 100x SYBR Gold (SG, 1\% dilution from 10000x stock) as a nuclear stain [21] and $20 \mu \mathrm{g} / \mathrm{ml}$ sulforhodamine 101 (SR) as a counterstain dissolved in 70\% ethanol. This is followed by a 30 second rinse in PBS to wash off excess stain. Stained specimens were then placed against a $200 \mu \mathrm{m}$ thick quartz coverslip attached to a histology cassette as an optical window. Each specimen was padded with PBS-soaked histology foam to provide compression and prevent dehydration. For evaluating the optical sectioning thickness, fixed mouse muscles from C57BL/6J mice from protocol approved by University of Rochester Committee on Animal Research (UCAR) were stained only with 100x SYBR Gold and rinsed for 30 seconds with PBS.

\subsection{Surgical specimens and fluorescence staining}

We designed an objective dipping cap that incorporates high-incidence angle illumination close to the tissue sample shown in the CAD model in Fig. 1(a). This 3D printed part mounts three evenly spaced $280 \mathrm{~nm} 16 \mathrm{~mW}$ LEDs (VPS164, Nikkiso) which couples into sapphire waveguides (SP-24A, Swiss Jewel). At the back of the LED boards are thermal epoxied heatsinks. These illuminator assemblies are directed at a $70^{\circ}$ angle of incidence towards the quartz coverslip relative to the surface normal. The 3D printed part is mounted a fixed distance above the quartz coverslip which allows for embedding a thin layer of immersion fluid such as glycerol $(\mathrm{n}=1.529$ at $280 \mathrm{~nm}$ ) in between the dipping cap and the quartz coverslip. This objective dipping cap remains static as the objective translates along the $\mathrm{z}$-axis to change focus.

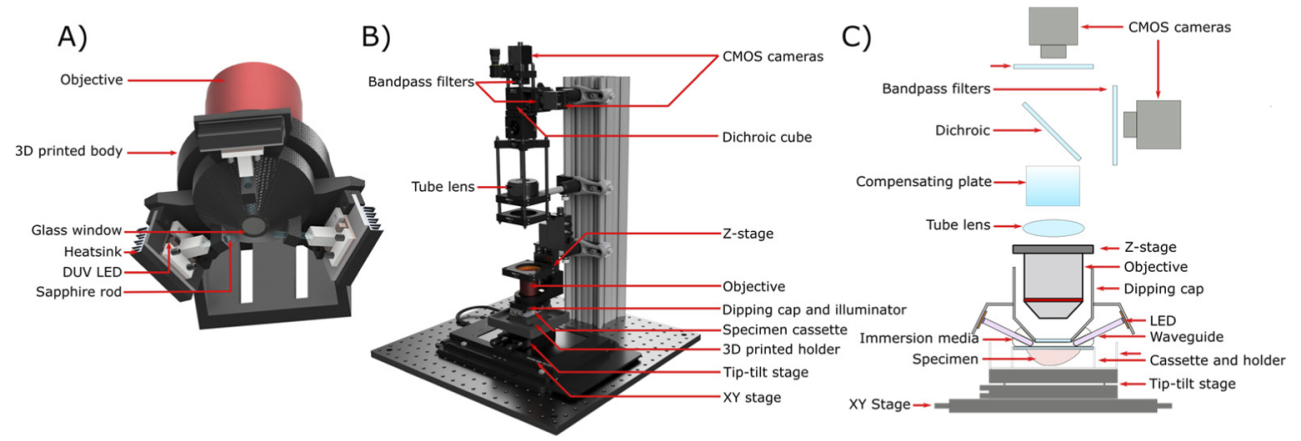

Fig. 1. a) Model of the 3D printed dipping cap and waveguide-based illuminator. b) Model of the whole MUSE system. c) 2D schematic of the MUSE system. CAD models are available here: https://github.com/vchingroa/MuseIlluminator

\subsection{MUSE system setup}

A model of our dual channel fluorescence MUSE system is shown in Fig. 1(b). The specimen cassette is mounted onto a motorized XY stage (MLS203-1, Thorlabs) which allow for tiling mosaic images by translating the specimen. The fluorescence signal from the UV-illuminated specimen is collected with a $4 \times 0.28 \mathrm{NA}$ air objective (XLFLUOR4X/340, Olympus) and a tube 
lens (TTL200, Thorlabs) and then relayed onto a dichroic mirror (T588lpxr-UF1, Chroma) which divides the visible fluorescence signal into two channels. Prior to this dichroic, we introduce transmissive glass matching the dichroic thickness oriented perpendicular to the dichroic to reduce astigmatism as per the nodal aberration theory and lens simulations. The first channel uses an optical bandpass filter with 600-680 nm passband (ET640/80, Chroma) to capture fluorescence signal from SR $\left(\lambda_{\text {em,peak }}=605 \mathrm{~nm}\right)$ while the second channel uses a bandpass filter with 518$558 \mathrm{~nm}$ (FF01-538/40, Semrock) to capture fluorescence signal from $S G\left(\lambda_{\text {em,peak }}=539 \mathrm{~nm}\right)$. Both channels are detected by two 20 MP CMOS cameras (BFS-U3-200S6M-C, FLIR). A motorized z-axis stage (X-LSM025A, Zaber) allows for depth scanning by translating the objective relative to the specimen. Resulting image frames from a single acquisition have a field of view of $3 \mathrm{~mm} \times 2 \mathrm{~mm}$. The XY stage mounts a tip-tilt stage for surface orientation correction such that the coverslip is completely normal to the optical axis. Mounted on the tip-tilt stage is a 3D printed cassette holder with lipped edges which prevents spillage of immersion media down the translational stage.

\subsection{Imaging and post-processing}

Image acquisition was automated using custom software written in Visual C++. While glycerol immersion required only a $\sim 25-50 \mathrm{~ms}$ exposure time, images were acquired with an integration time of $400 \mathrm{~ms}$ due to lower illumination efficiency with air immersion. Tile images were acquired with $1.5 \mathrm{~mm}$ spacing from each other to ensure significant overlap for channel coregistration and stitching. Depth stacking was performed with a step size of $3 \mu \mathrm{m}$ over a range of up to $50 \mu \mathrm{m}$ to capture information for focal stacking. The following post-processing steps were performed in MATLAB. First, dark frame subtraction and channel crosstalk subtraction were applied to individual frames. The two fluorescence channels for each frame were coregistered by applying an affine transformation to account for camera alignment offsets. Pre-processed image z-stacks were then deconvolved using a GPU-accelerated 3D Richardson-Lucy algorithm (YacuDecu [26]). The PSF was measured by imaging $300 \mathrm{~nm}$ Europium carboxylate beads (Fluoresbrite, Polysciences). Deconvolved image z-stacks were focal stacked using an open-source exposure fusion algorithm (Enfuse [27]) resulting in individual images with extended depth of field. These images were then stitched using Image Composite Editor (Microsoft) into full mosaics and rendered into virtual H\&E using a previously developed coloring algorithm [28].

\subsection{Evaluation of image quality and optical sectioning thickness}

To evaluate image improvements resulting from high-index immersion illumination, a frozen skin excision was imaged first with air immersion, then with water and finally with glycerol. Afterwards, the sample was fixed and was submitted for paraffin histology. Resolution was measured through the 10-90 step response using the edge of a solidly stained nucleus. Contrast was calculated with $\left(I_{\max }-I_{\min }\right) /\left(I_{\max }+I_{\min }\right)$ across the linear profile [21].

For evaluating optical sectioning thickness, fixed mouse muscle tissue was imaged with each immersion medium. Muscle tissue was selected because the small size and sparse positioning of nuclei makes them straightforward to segment. Tissue was then volumetrically imaged using a two-photon excitation fluorescence microscope $\left(\lambda_{\mathrm{ex}}=780 \mathrm{~nm}\right)$ with detection bands of 534/30 for SG. The depths of each nucleus were measured using the two-photon dataset. MUSE images with air, water and glycerol immersion were coregistered with the two-photon image. The signal intensity from the MUSE images was then mapped to the depth information from the two-photon images set similar to the work of Yoshitake et al. [19]. While individual nuclei have varying brightness, by averaging over a statistically large number of nuclei, the mean MUSE intensities will show an exponential decrease with increasing depths, following Beer's law. Particularly, the detected MUSE fluorescence $I=\phi I_{o} e^{-\left(\mu_{t, 280}+\mu_{t, 539) d}\right.}+I_{b g}$ where $\phi$ is the quantum yield, $I_{0}$ is the incident excitation, $u_{t, \lambda}$ is the total attenuation coefficient at wavelength $\lambda, d$ is depth, and 
$I_{b g}$ is the background signal. As such, an exponential fit with a background term is used, and the normalized $\mathrm{e}^{-1}$ depth or the mean free path is used as a measure of the optical sectioning thickness.

For evaluating cellularity with cell counting, another frozen skin excision was imaged with water immersion and then with glycerol immersion. Nuclear channels of a region were processed with ImageJ as follows for cell segmentation: 1) intensity normalization, 2) contrast-limited adaptive histogram normalization (CLAHE), 3) mask generation through thresholding, 4) watershed segmentation, and 5) cell counting.

\section{Results}

\subsection{Theory, simulation, and design for illumination immersion}

Angled illumination offers a way to reduce the optical sectioning thickness as shown in previous MUSE systems [15,19,21]. Illumination normal to the tissue surface (Fig. 2(a)) excites 63.2\% of total fluorescence within a section that is one mean free absorption path deep. With angled illumination (Fig. 2(b)), the incident DUV photons still travel, on average, one mean free absorption path before absorption but since the path is angled relative to the tissue surface, the optical sectioning thickness becomes the tangential component of the mean free absorption path. Increasing the angle of illumination therefore reduces optical section thickness. However, the maximum angle of refraction at the air-to-tissue interface is limited by refraction as shown in Fig. 2(d), with high-index immersion media required to reach angles approaching $90^{\circ}$ as shown in Fig. 2(c). Moreover, the use of a high-index immersion media offers practical advantages in tissue illumination efficiency. Because less steep illumination angles can be used to achieve very high illumination angle (after refraction), the extent of the illumination pattern projected onto the tissue surface plane is more circular and less elongated and therefore less light is illuminating areas outside the objective field of view. Additionally, high-index immersion media that are closer to the refractive index of the quartz window have less reflection loss which again leads to higher tissue illumination efficiency.

These advantages from high-index immersion angled illumination are more concretely demonstrated through ray-tracing simulations. Using the specified radiant intensity of our DUV LED, we simulate our LED coupled into a waveguide angled at $70^{\circ}$ pointed at a quartz window $(n=1.4924)$ prior to the tissue $(n=1.4)$. In three different scenarios, we immersed the LED waveguides in air $(n=1)$, water $(n=1.353)$, and glycerol $(n=1.529)$ and simulated the relative angular intensity distribution across the tissue surface as shown in Fig. 2(e). We also calculated the relative theoretical optical sectioning thickness improvements for both according to the weighted average of the angles of refraction, as well as calculated the relative illumination flux over the objective field of view as listed in Table 1. We note that even with high index immersion, the average refracted angle is lower than expected due to loss of higher angle rays to total internal reflection, a loss which could be improved through further optimization of illumination angle and/or refractive index.

Table 1. Effects of refractive index of the illumination immersion media

\begin{tabular}{clll}
\hline & Air $(\mathrm{n}=1)$ & Water $(\mathrm{n}=1.353)$ & Glycerol $(\mathrm{n}=1.529)$ \\
\hline${\overline{\theta_{i}}}^{a}$ & $70^{\circ}$ & $70^{\circ}$ & $70^{\circ}$ \\
${\overline{\theta_{r}}}^{a}$ & $33^{\circ}$ & $55^{\circ}$ & $69^{\circ}$ \\
Relative sectioning thickness & 1 & 0.68 & 0.43 \\
Relative illumination flux & 1 & 1.91 & 1.86 \\
\hline
\end{tabular}

${ }^{a}$ Weighted average of angles of incidence and refraction for assumed tissue refractive index of 1.4 


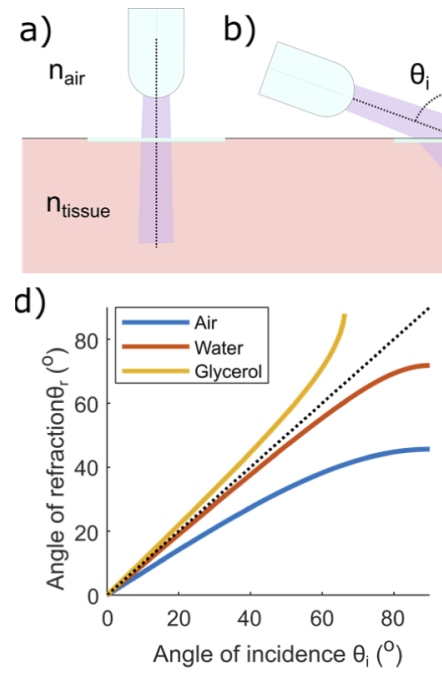

c) م
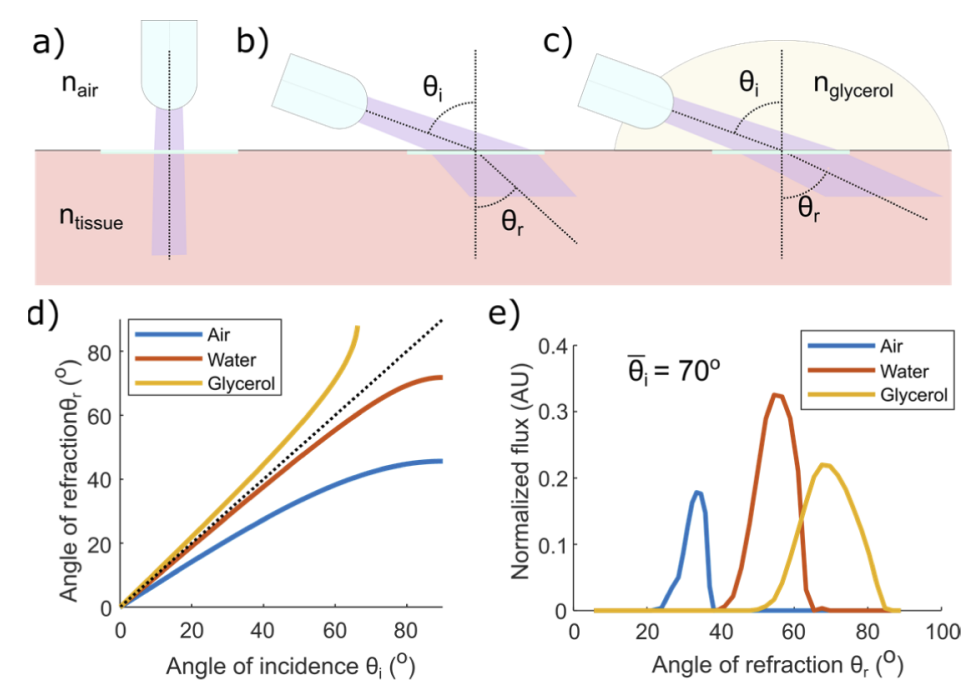

Fig. 2. MUSE illumination into a quartz coverslip and onto the tissue with a) normal illumination. b) angled illumination. c) angled illumination with immersion media. d) Angle of refraction into the tissue as a function of angle of incidence with air, water, and glycerol immersion. Dotted line represents $\theta_{i}=\theta_{r}$. e) Relative angular intensity distribution of DUV illumination across the tissue surface for different illumination immersion media with mean angle of incidence $\bar{\theta}_{i}=70^{\circ}$

Water immersion illumination has been implemented previously through the use of immersion objectives by Yoshitake et al. [19]. However, this approach couples both illumination parameters (angle of illumination, immersion media) and detection parameters (objective numerical aperture, field of view). This can be challenging in practice; for example, reducing the optical sectioning thickness to the same degree as glycerol in Table 1 restricts the choice among commercially available objectives to oil or other high index immersion objectives which typically have very narrow fields of view. Conversely, a large field of view restricts one to air objectives where the optical sectioning thickness is large with MUSE. Our design is therefore driven by the idea of decoupling illumination and detection parameters which allow for high field of view, low optical sectioning thickness MUSE with commercially available low magnification air objectives and high-index immersion media. This was implemented using an objective dipping cap design as shown in Fig. 1(f). where a thin layer $(<2 \mathrm{~mm})$ of immersion media can be embedded between the dipping cap window and the quartz specimen window. The output ends of the waveguides are close to the specimen window and are submerged into the thin immersion media for immersed angled illumination. Overall, we achieve a $6 \mathrm{~mm}^{2}$ field of view with a depth of field of $\sim 10 \mu \mathrm{m}$.

\subsection{Reduction in optical sectioning thickness and background fluorescence}

The use of an immersion dipping cap enables the same objective to be used with immersion media of any refractive index. We exploit this freedom to measure the reduction in optical sectioning thickness as a function of immersion media refractive index for air, water, and glycerol as simulated in Table 1. Figure 3(a)-(c) shows MUSE images for three different immersion media. First, the reduction in cellularity is visually apparent going from index of air towards glycerol. Looking at a single cell at $18 \mu \mathrm{m}$ depth (red boxes), fluorescence from the nuclei is attenuated going from air to water to glycerol with the zoomed-in regions (Fig. 3(d)-(f)) showing decreasing contrast from background until it disappears entirely in high index glycerol MUSE. This is shown more apparent in the intensity profiles shown in Fig. $3(\mathrm{~g})$. For $70^{\circ}$ incidence 
angle, illumination with air resulted in a $20.28 \mu \mathrm{m}(18.60-22.30 \mu \mathrm{m}, 95 \% \mathrm{CI})$ optical section, while water and glycerol resulted in $10.43 \mu \mathrm{m}(9.60-11.43 \mu \mathrm{m}, 95 \% \mathrm{CI})$ and $6.67 \mu \mathrm{m}(6.09-7.37$ $\mu \mathrm{m}, 95 \% \mathrm{CI}$ ), respectively. Relative to air, the reduction in sectioning thickness due to water and glycerol is 0.51 and 0.33 , respectively, compared to the simulated 0.68 and 0.43 reduction. Additionally, we observe a 4\% decrease in the uniform background fluorescence relative to air, measured as the minimum baseline of the exponential decrease, due at least in part to reduced scattered fluorescence from deeper regions.

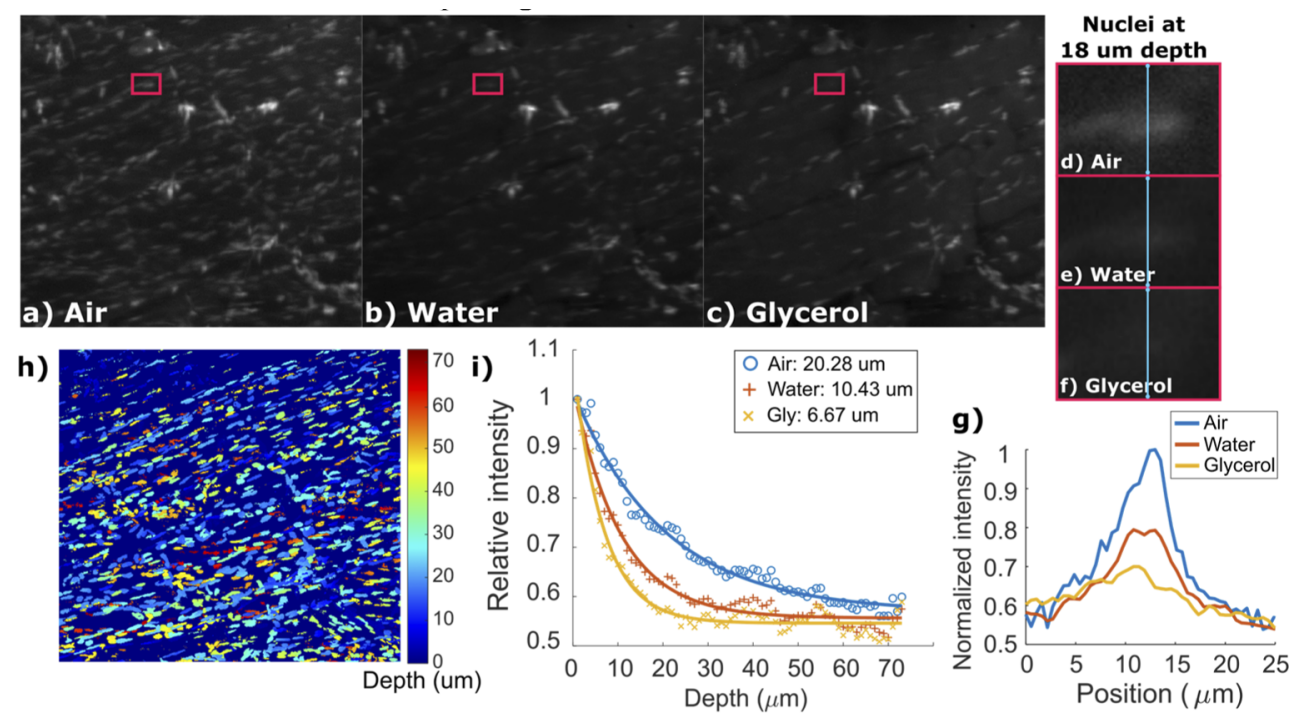

Fig. 3. MUSE images of the same region of mouse muscle specimen with a) air, b) water, and c) glycerol illumination immersion. Red boxes enclose a cell at $18 \mu \mathrm{m}$ depth with zoomed in regions at respective immersions (d-f) enclosed in a red box. $g$ ) Intensity profiles of the cyan lines in (d-f). h) Segmented height map of cells from volumetric data acquired with two-photon excitation microscopy. i) Average intensities of cells at each depth for air, water and glycerol illumination immersion with corresponding exponential fitting following Beer's law.

\subsection{Increased contrast and depth of field with post-processing}

The detection optical path goes through a thin layer of immersion media and quartz coverslip which induces optical aberrations, primarily spherical aberration. By measuring the PSF with fluorescent beads (Fig. 4(a)), we deconvolved image stacks using the 3D Richardson-Lucy algorithm to compensate for aberrations and improve resolution and contrast as shown in Fig. 4(b)-(c), with example 1D profiles shown in Fig. 4(d). Here we show around 7\% improved resolution based on step response, and 35\% improved contrast. Additionally, tissue excisions typically have substantial surface irregularities that cannot be completely resolved under one focal volume. Therefore, focal stacking, which extends the depth-of-field, was performed after deconvolution to reconstruct a 2D mosaic that is completely resolved despite surface irregularities. Effects of this are shown in Fig. 4(e)-(f) wherein a region that is out of focus from the focal plane of the bulk of the tissue (Fig. 4(e)) is better resolved in the focal stacked image (Fig. 4(f)). Finally, full improvements for both deconvolution and focal stacking is shown in Fig. 4 g. 

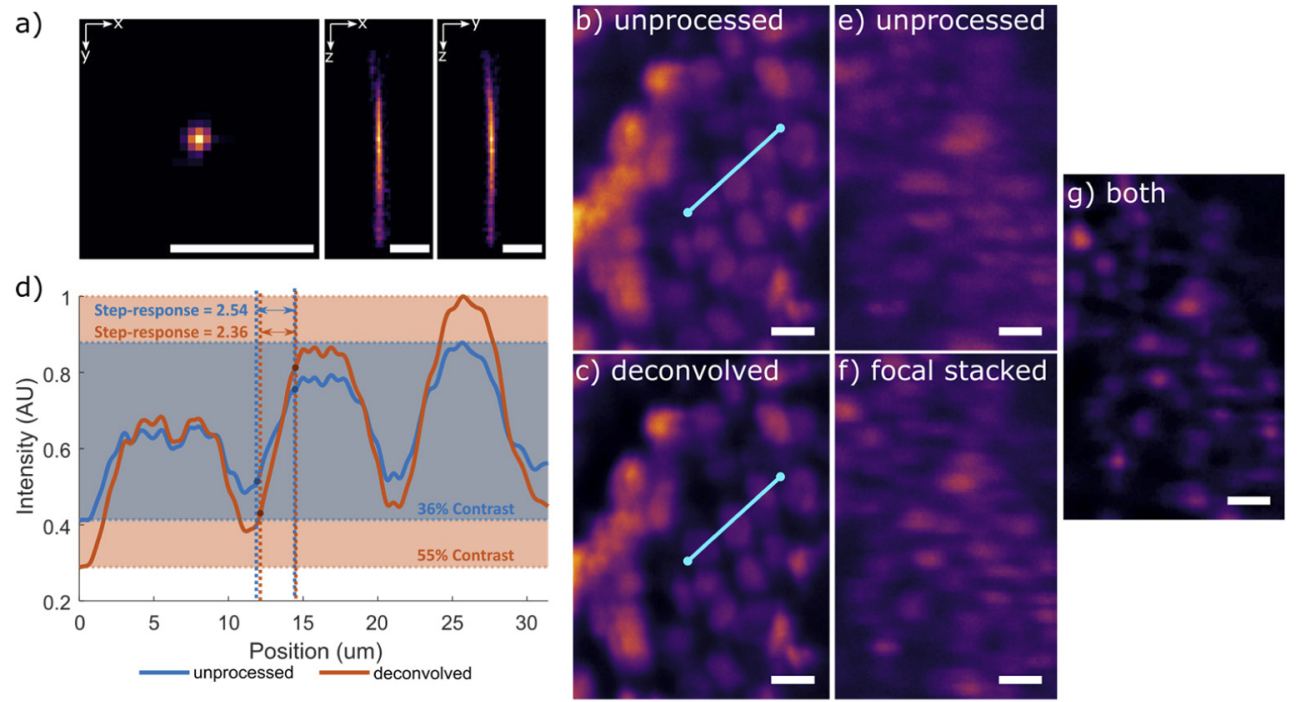

Fig. 4. a) Orthogonal cross-section views of the point spread function measured from $300 \mathrm{~nm}$ Europium carboxylate beads. b) A non-deconvolved image region from a full tissue mosaic and c) its matching deconvolved image. d) Intensity profiles of the cyan lines from $b$ and $c$ showing step responses (from nuclei edge) and contrast of unprocessed and deconvolved images. e) An out-of-focus image region of a full mosaic, f) the same region focus-stacked, and g) the same region deconvolved and focus-stacked. Scalebars: $10 \mu \mathrm{m}$

\subsection{Reduced feature density and cellularity compared with paraffin sections}

To evaluate correspondence with conventional paraffin sections, a skin excision was imaged with air and glycerol immersion MUSE, and then subsequently fixed and submitted for paraffin processing. Full tissue mosaics of a skin biopsy and zoomed in images of a hair bulb with air and glycerol immersion MUSE and H\&E paraffin sections are shown in Fig. 5(a)-(c). A hair bulb is enclosed with interfollicular epidermis and bulge cells [29], which in $5 \mu \mathrm{m}$ paraffin sections show as a few-cell-thick layer outlining the keratinous cortex (Fig. 5(d)-(f), green boxes). In contrast, with thicker optical sectioning, the cells underlying the acellular hair follicle are captured resulting in a denser-appearing cell layer in the air immersion MUSE images. Reducing the optical sectioning thickness with glycerol immersion results in the attenuation of signal from these deeper cells under the bulb and a reduced cellularity. The magnified region of the cell layer shown in Fig. 5(g)-(i) show cells (red arrows) that are not present in H\&E paraffins. Four of these cells are completely visible in air immersion MUSE, while half of them are heavily attenuated in glycerol MUSE, and the other half are completely attenuated. Cells that are present in the H\&E paraffins (cyan arrows) are still visible with both MUSE immersions. Such cellularity differences are still apparent without the gamma compression associated with virtual H\&E rendering as shown in the magnified nuclear channels in Fig. 5(j)-(k). Cluster of cells that are similarly underneath the acellular hair follicle and therefore are not captured by H\&E paraffin section (Fig. 5(n)) are heavily attenuated or not captured in glycerol immersion MUSE (Fig. 5 m, red arrows) while being significantly brighter in air immersion MUSE (Fig. 51, red arrows). Nuclear channels without gamma compression show the same apparent difference with Fig. 5(o)-(p). Qualitatively, these result in a more similar layer thickness and structure as with the paraffin sections. Additionally, the reduction in background levels as shown in Fig. 3, is more apparent here through virtual H\&E coloration (exponential mapping) when comparing air and glycerol immersion MUSE. 


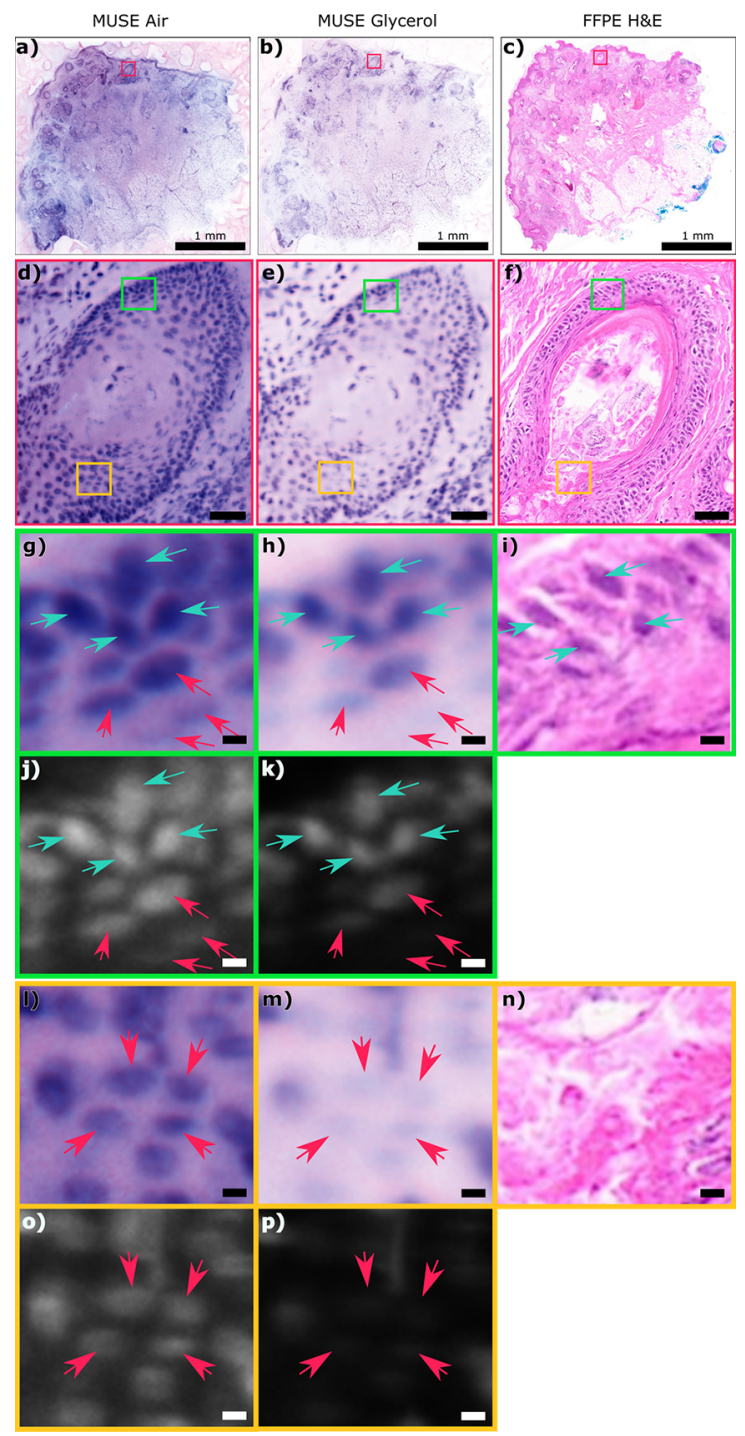

Fig. 5. Virtual H\&E mosaics of a skin biopsy using a) air immersion MUSE (https://imstore.circ.rochester.edu/papers/muse/Fig5/Fig5a/air.html) and b) glycerol immersion MUSE (https://imstore.circ.rochester.edu/papers/muse/Fig5/Fig5b/glycerol.html). c) Brightfield $\mathrm{H} \& \mathrm{E}$ mosaic of an FFPE histology section (https://imstore.circ.rochester.edu/papers/muse/Fig5/Fig5c/ffpe.html). Zoomed in region showing a hair bulb from c) air immersion MUSE mosaic, d) glycerol immersion MUSE mosaic and e) H\&E paraffin section, with green boxes showing a region of a bulge cell layer and orange boxes showing a region of acellular hair follicle. Zoomed in region of the green boxes are shown for $\mathrm{g}$ ) air immersion MUSE, $\mathrm{h}$ ) glycerol immersion MUSE, and i) $H \& E$ paraffin section, with cyan arrows labeling cells present in the paraffin section and red arrows labeling cells absent. Nuclear channels of g) and h) without gamma correction are shown in $\mathrm{j}$ ) and k) respectively. Zoomed in region of the orange boxes are shown for l) air immersion MUSE, m) glycerol immersion MUSE, and n) H\&E paraffin section with red arrows labeling cells absent in the paraffin section. Nuclear channels of 1) and m) without gamma correction are shown in o) and p). Scalebars: (a-c) $1 \mathrm{~mm}$ and (d-f) $50 \mu \mathrm{m}$ (g-p) $5 \mu \mathrm{m}$ 
We also show significant improvements in cellularity with skin biopsies when going from water immersion to glycerol immersion. A region of dense inflammation around basal cell carcinoma (Fig. 6(a)-(b), red boxes), show significantly increased cellularity from water immersion MUSE compared to glycerol immersion MUSE due to capturing deeper nuclei. Analyzing the raw nuclear channels (Fig. 6(c)-(d)) and performing cell segmentation (Fig. 6(e)-(f)) reveal a decrease in the number of cells captured from 453 to 367 going from water to glycerol immersion ( 19\% decrease). This also equates to a $\sim 33 \%$ reduction in total fraction of images occupied by nuclei.
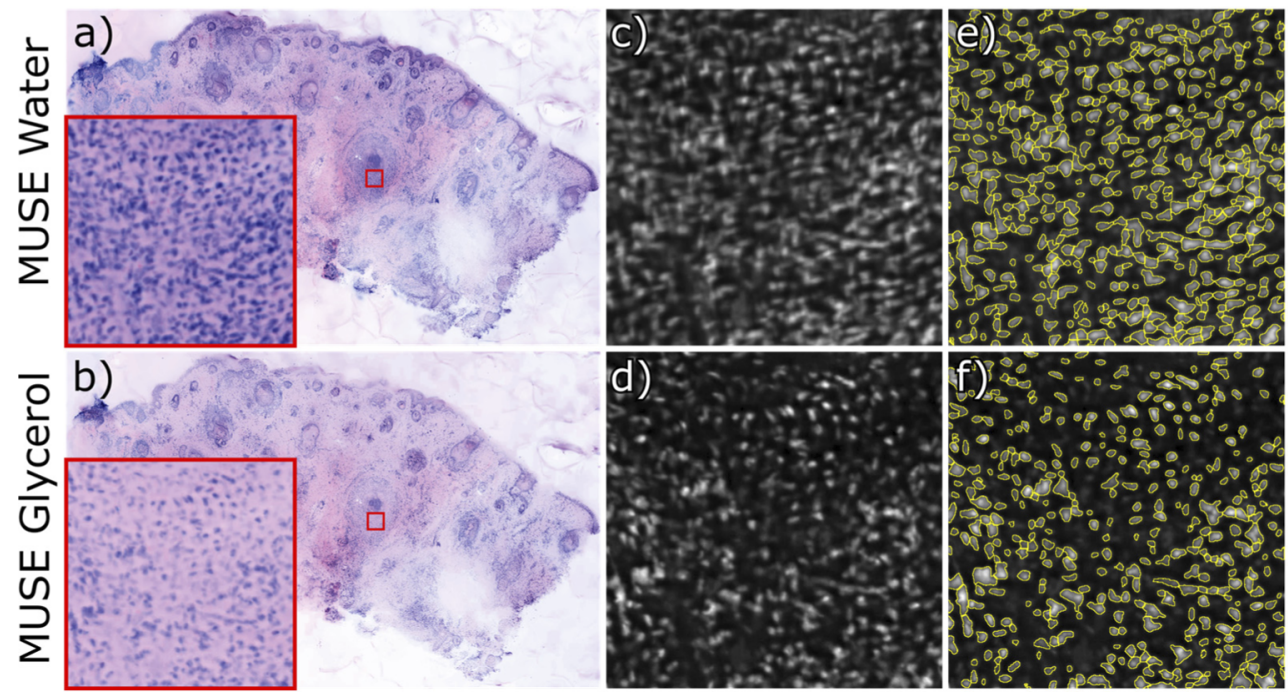

Fig. 6. Virtual H\&E mosaics of a skin biopsy using a) water immersion MUSE (https://imstore.circ.rochester.edu/papers/muse/Fig6/Fig6a/water.html) and b) glycerol immersion MUSE (https://imstore.circ.rochester.edu/papers/muse/Fig6/Fig6b/glycerol.html) with zoomed-in regions shown in red boxes of inflammation around basal cell carcinoma. Nuclear channels without virtual H\&E gamma correction for c) water immersion MUSE and d) glycerol immersion MUSE. Segmented nuclear channels for e) water immersion MUSE and f) glycerol immersion MUSE with total cell count of 453 and 367 respectively.

\section{Discussion}

MUSE has attracted attention as an alternative to paraffin and frozen sections, especially for low-cost histology applications. However, the thicker optical sectioning provided by MUSE compared to conventional paraffin sections results in decreased contrast and the appearance of increased cellularity as compared to conventional histology. A previous attempt to reduce this optical sectioning thickness used immersion objectives. While theory suggests that further increasing immersion refractive index will further reduce optical sectioning thickness, practical implementations of such systems are difficult. High index immersion objectives are costly, and are almost entirely very high magnification while much of histological practice relies on low magnification for larger field of view. We address this by designing a 3D printed illuminator and objective dipping cap that decouples illumination from detection parameters. This allows the use of large field of view air objectives for higher image throughput and real-time evaluation while enabling improved optical sectioning through high index immersion. While we used glycerol $(n=1.529)$ due to its low cost and ease of use, other higher index immersion fluids and/or more optimized illumination angles could be used to further improve resolution and contrast. Our simulations (Fig. 2) show a substantial contribution from rays striking the sample at less than 60 
degrees incidence, suggesting that further improvements in illuminator design or higher index immersion could further improve optical sectioning.

Further reductions in optical section thickness would have several advantages. First, in MUSE, the optical section thickness is analogous to axial resolution in other forms of microscopy, thus images are directly improved. Second, optical sectioning thickness above $5 \mu \mathrm{m}$ results in histological images appearing more cellular than conventional histology using $5 \mu \mathrm{m}$ sections, complicating image interpretation in medical applications. Finally, to avoid blurring from out of focus light, the depth of field of the objective should be greater than the optical section thickness, thus improvements are especially advantageous with higher numerical aperture objectives, for which the depth of focus would be less than the MUSE optical section thickness in air or water.

The main disadvantage of the dipping cap approach is the induced spherical aberration from the immersion media. However, as MUSE optical sectioning thickness is independent of the imaging system, this has no effect on optical sectioning and only contributes to lateral blurring. Furthermore, due to the limited NA typically used with MUSE, this induced spherical aberration has a limited effect on lateral resolution, with no increase in full width half maximum width of the point spread function and only a modest increase side lobe energy (Strehl ratio decrease to $\sim 0.52$ for an ideal $0.28 \mathrm{NA}$ objective). In this work, deconvolution was used to minimize the effect of the higher sidelobes. Although high NA objectives are difficult to use with MUSE due to limited depth of field, optical approaches such as using objectives with a correction collar could also be employed. These approaches should enable variable objective MUSE with both high field-of-view, low numerical aperture objectives for large area surveyance and high magnification and numerical aperture objectives for examining cellular characteristics, similar to current imaging workflows in conventional histopathology. Similarly, while we employ focal stacking to extend depth of field, we rely on extended depth of field only for small regions of surface irregularity and not for negating the effects of depth of field and optical sectioning thickness mismatch. This enables viewing the specimen in real-time analogously to a conventional histology microscope without acquiring and processing the full z-stack for focal-stacking.

To our knowledge, this is the first demonstration of a higher-than-water refractive index medium used for MUSE immersion. Our quantitative measurements of the MUSE optical sectioning thicknesses show a $\sim 36 \%$ reduction in optical sectioning thickness compared to water $(67 \%$ reduction compared to air) immersion as well a modest improvement in contrast and reduction in background fluorescent signal. In addition, our measurements of the axial section thickness for water and air are consistent with those published previously without using a dipping cap [19], confirming that our approach performs similarly while enabling higher index immersion. Finally, these results are confirmed by cell counting, which show proportional reductions in the fraction of tissue occupied by nuclei, and overall density of nuclei that are much closer to typical paraffin sections than comparable water or air immersion MUSE measurements. We note that due to the unique dipping cap design, our approach can utilize conventional air objectives while still improving optical sectioning thickness. Thus, in contrast to previous work using dipping objectives, glycerol immersion can be incorporated into MUSE systems while utilizing existing objectives and imposing no reduction in field of view.

The rapidly developing market for DUV LEDs has led to increasingly powerful and inexpensive DUV LEDs. These dramatic decreases in cost may make more powerful illuminators and proportionally higher imaging speeds feasible with minor iterations (e.g., 6 LED ring design) of the illuminator presented here. To encourage further experimentation, we have made our MUSE illuminator CAD files freely available at https://github.com/vchingroa/Musellluminator. While acquisition frame rates were not the focus of this work, higher illumination power will directly enable proportionate reductions in acquisition times, enabling higher throughput imaging. 


\section{Conclusion}

We demonstrate that the use of high-index immersion with a dipping cap is a cost-effective way to significantly improve the optical sectioning capability of MUSE. We show that glycerol immersion results in improved contrast and reduced appearance of cellularity in MUSE images and can be performed without requiring higher magnification water or oil immersion objectives. Additionally, deconvolution and focus stacking improve MUSE resolution and enables robust imaging of irregular tissue surfaces.

Funding. U.S. National Institutes of Health (K22-CA226035-03, R01 CA258376-01).

Acknowledgments. We would like to thank the Histology, Biochemistry and Molecular Imaging (HBMI) core for helping with FFPE histology.

This study was supported by the U.S. National Institutes of Health, Grant No. K22-CA226035-03 and R01 CA258376-01. The Histology, Biochemistry and Molecular Imaging (HBMI) core was supported by NIH AR069655.

Disclosures. The authors declare no conflicts of interest.

Data availability. Data underlying the results presented in this paper are not publicly available at this time but may be obtained from the authors upon reasonable request.

\section{References}

1. S. J. Schnitt and M. Morrow, "Should intraoperative frozen section evaluation of breast lumpectomy margins become routine practice?" Am. J. Clin. Pathol. 138(5), 635-638 (2012).

2. V. Rastogi, N. Puri, S. Arora, G. Kaur, L. Yadav, and R. Sharma, “Artefacts: A diagnostic dilemma - A review," J. Clin. Diagn. Res. 7, 2408-2413 (2013).

3. C. Longo, M. Ragazzi, M. Rajadhyaksha, K. S. Nehal, A. Bennàssar, G. Pellacani, and J. Malvehy Guilera, "In Vivo and Ex Vivo Confocal Microscopy for Dermatologic and Mohs Surgeons," Dermatol. Clin. 34(4), 497-504 (2016).

4. N. V. Iftimia, G. Peterson, E. W. Chang, G. Maguluri, W. Fox, and M. Rajadhyaksha, "Combined reflectance confocal microscopy-optical coherence tomography for delineation of basal cell carcinoma margins: an ex vivo study," J. Biomed. Opt. 21(1), 016006 (2016)

5. S. Abeytunge, Y. Li, B. Larson, G. Peterson, E. Seltzer, R. Toledo-Crow, and M. Rajadhyaksha, "Confocal microscopy with strip mosaicing for rapid imaging over large areas of excised tissue,” J. Biomed. Opt. 18(6), 061227 (2013).

6. L. C. Cahill, Y. Wu, T. Yoshitake, C. Ponchiardi, M. G. Giacomelli, A. A. Wagner, S. Rosen, and J. G. Fujimoto, "Nonlinear microscopy for detection of prostate cancer: analysis of sensitivity and specificity in radical prostatectomies," Mod. Pathol. (2019).

7. M. G. Giacomelli, T. Yoshitake, L. C. Cahill, H. Vardeh, L. M. Quintana, B. E. Faulkner-Jones, J. Brooker, J. L. Connolly, and J. G. Fujimoto, "Multiscale nonlinear microscopy and widefield white light imaging enables rapid histological imaging of surgical specimen margins," Biomed. Opt. Express 9(5), 2457 (2018).

8. M. G. Giacomelli, B. E. Faulkner-Jones, L. C. Cahill, T. Yoshitake, D. Do, and J. G. Fujimoto, "Comparison of nonlinear microscopy and frozen section histology for imaging of Mohs surgical margins," Biomed. Opt. Express 10(8), 4249-4260 (2019).

9. E. Olson, M. J. Levene, and R. Torres, "Multiphoton microscopy with clearing for three dimensional histology of kidney biopsies," Biomed. Opt. Express 7(8), 3089 (2016).

10. C. W. Freudiger, R. Pfannl, D. A. Orringer, B. G. Saar, M. Ji, Q. Zeng, L. Ottoboni, W. Ying, C. Waeber, J. R. Sims, P. L. De Jager, O. Sagher, M. A. Philbert, X. Xu, S. Kesari, X. S. Xie, and G. S. Young, "Multicolored stain-free histopathology with coherent Raman imaging,” Lab. Invest. 92(10), 1492-1502 (2012).

11. S. Luethy, D. B. Tulman, and J. Q. Brown, "Automated gigapixel circumferential surface microscopy of the prostate," Sci. Rep. 10(1), 131-214 (2020).

12. A. K. Glaser, N. P. Reder, Y. Chen, C. Yin, L. Wei, S. Kang, L. A. Barner, W. Xie, E. F. McCarty, C. Mao, A. R. Halpern, C. R. Stoltzfus, J. S. Daniels, M. Y. Gerner, P. R. Nicovich, J. C. Vaughan, L. D. True, and J. T. C. Liu, "Multi-immersion open-top light-sheet microscope for high-throughput imaging of cleared tissues," Nat. Commun. 10(1), 2781 (2019).

13. N. P. Reder, A. K. Glaser, E. F. McCarty, Y. Chen, L. D. True, and J. T. C. Liu, "Open-top light-sheet microscopy image atlas of prostate core needle biopsies," Arch. Pathol. Lab. Med. 143(9), 1069-1075 (2019).

14. F. Fereidouni, A. Datta-Mitra, S. Demos, and R. Levenson, "Microscopy with UV surface excitation (MUSE) for slide-free histology and pathology imaging," Opt. Biopsy XIII Towar. Real-Time Spectrosc. Imaging Diagnosis 9318 93180F (2015).

15. F. Fereidouni, Z. T. Harmany, M. Tian, A. Todd, J. A. Kintner, J. D. McPherson, A. D. Borowsky, J. Bishop, M Lechpammer, S. G. Demos, and R. Levenson, "Microscopy with ultraviolet surface excitation for rapid slide-free histology," Nat. Biomed. Eng. 1(12), 957-966 (2017).

16. S. Demos and R. Levenson, "System and method for controlling depth of imaging in tissues using fluorescence microscopy under ultraviolet excitation following staining with fluorescing agents.," U.S. patent US9625387B2 (2017). 


\section{Biomedical Optics EXPRESS}

17. F.-X. Schmid, "Biological Macromolecules: UV-visible Spectrophotometry," in Encyclopedia of Life Sciences (John Wiley \& Sons, Ltd, 2001), Vol. 99, pp. 178-181.

18. A. Grau, F. Guardiola, J. Boatella, M. D. Baucells, and R. Codony, "Evaluation of lipid ultraviolet absorption as a parameter to measure lipid oxidation in dark chicken meat," J. Agric. Food Chem. 48(9), 4128-4135 (2000).

19. T. Yoshitake, M. G. Giacomelli, L. M. Quintana, H. Vardeh, C. Lucas, B. E. Faulkner-jones, J. L. Connolly, D. Do, and J. G. Fujimoto, "Rapid histopathological imaging of skin and breast cancer surgical specimens using immersion microscopy with ultraviolet surface excitation," Sci. Rep. 8(1), 4476 (2018).

20. T. Matsumoto, H. Niioka, Y. Kumamoto, J. Sato, O. Inamori, R. Nakao, Y. Harada, E. Konishi, E. Otsuji, H. Tanaka, J. Miyake, and T. Takamatsu, "Deep-UV excitation fluorescence microscopy for detection of lymph node metastasis using deep neural network," Sci. Rep. 9(1), 16912 (2019).

21. W. Xie, Y. Chen, Y. Wang, L. Wei, C. Yin, A. K. Glaser, M. E. Fauver, E. J. Seibel, S. M. Dintzis, J. C. Vaughan, N. P. Reder, and J. T. C. Liu, "Microscopy with ultraviolet surface excitation for wide-area pathology of breast surgical margins," J. Biomed. Opt. 24(02), 1 (2019).

22. T. Lu, J. M. Jorns, M. Patton, R. Fisher, A. Emmrich, T. Doehring, T. G. Schmidt, D. H. Ye, T. Yen, and B. Yu, "Rapid assessment of breast tumor margins using deep ultraviolet fluorescence scanning microscopy," J. Biomed. Opt. 25, 1-17 (2020).

23. E. R. Dowski and W. T. Cathey, "Extended depth of field through wave-front coding," Appl. Opt. 34(11), 1859 (1995).

24. L. Jin, Y. Tang, Y. Wu, J. B. Coole, M. T. Tan, X. Zhao, H. Badaoui, J. T. Robinson, M. D. Williams, A. M. Gillenwater, R. R. Richards-Kortum, and A. Veeraraghavan, "Deep learning extended depth-of-field microscope for fast and slide-free histology,” Proc. Natl. Acad. Sci. U. S. A. 117(52), 33051-33060 (2020).

25. Y. Liu, A. M. Rollins, R. M. Levenson, F. Fereidouni, and M. W. Jenkins, "Pocket MUSE: an affordable, versatile and high-performance fluorescence microscope using a smartphone," Commun, Biol. 4, 334 (2021).

26. B. Pepin, "GPU Deconvolution Library with C and Matlab API for nVidia GPUs," https://github.com/bobpepin/YacuDecu.

27. A. Mihal and C. Spiel, "Combining Multiple Images with Enfuse 4.2," http://enblend.sourceforge.net/enfuse.doc/enfuse_4.2.xhtml/enfuse.html.

28. M. G. Giacomelli, L. Husvogt, H. Vardeh, B. E. Faulkner-Jones, J. Hornegger, J. L. Connolly, and J. G. Fujimoto, "Virtual hematoxylin and eosin transillumination microscopy using epi-fluorescence imaging," PLoS One 11(8), e0159337 (2016).

29. P. Daszczuk, P. Mazurek, T. D. Pieczonka, A. Olczak, L. M. Boryń, and K. Kobielak, "An intrinsic oscillation of gene networks inside hair follicle stem cells: an additional layer that can modulate hair stem cell activities," Front. Cell Dev. Biol. 8, 1-21 (2020). 